Mappemonde

Revue trimestrielle sur l'image géographique et les

formes du territoire

$131 \mid 2021$

Varia

\title{
Passagers du ciel
}

Alice Caron

URL : https://journals.openedition.org/mappemonde/6329

DOI : 10.4000/mappemonde.6329

ISSN : 1769-7298

Éditeur

UMR ESPACE

Référence électronique

Alice Caron, «Passagers du ciel », Mappemonde [En ligne], 131 | 2021, mis en ligne le 08 juillet 2021,

consulté le 14 juillet 2021. URL : http://journals.openedition.org/mappemonde/6329 ; DOI : https:// doi.org/10.4000/mappemonde.6329

Ce document a été généré automatiquement le 14 juillet 2021.

\section{(c) (1)(2)}

La revue Mappemonde est mise à disposition selon les termes de la Licence Creative Commons Attribution - Pas d'Utilisation Commerciale - Partage dans les Mêmes Conditions 4.0 International. 


\section{Passagers du ciel}

\section{Alice Caron}

1 Ce texte vise à présenter les dessous de la production cartographique intitulée Passagers $d u$ ciel (figure 1). Cette œuvre visuelle originale a été réalisée dans le cadre du Mapathon proposé par le colloque Tous (im)mobiles, tous cartographes? (http:// cartomob.sciencesconf.org/). Elle respecte la contrainte de ne représenter que les données fournies par les organisateurs de l'événement et propose une histoire illustrée des flux aériens entre 1999 et 2019 via la cartographie. Le résultat de ce projet est axé sur l'évolution contrastée des liaisons aériennes depuis ou vers la Région AuvergneRhône-Alpes sur ces vingt dernières années.

Figure 1. Passagers du ciel

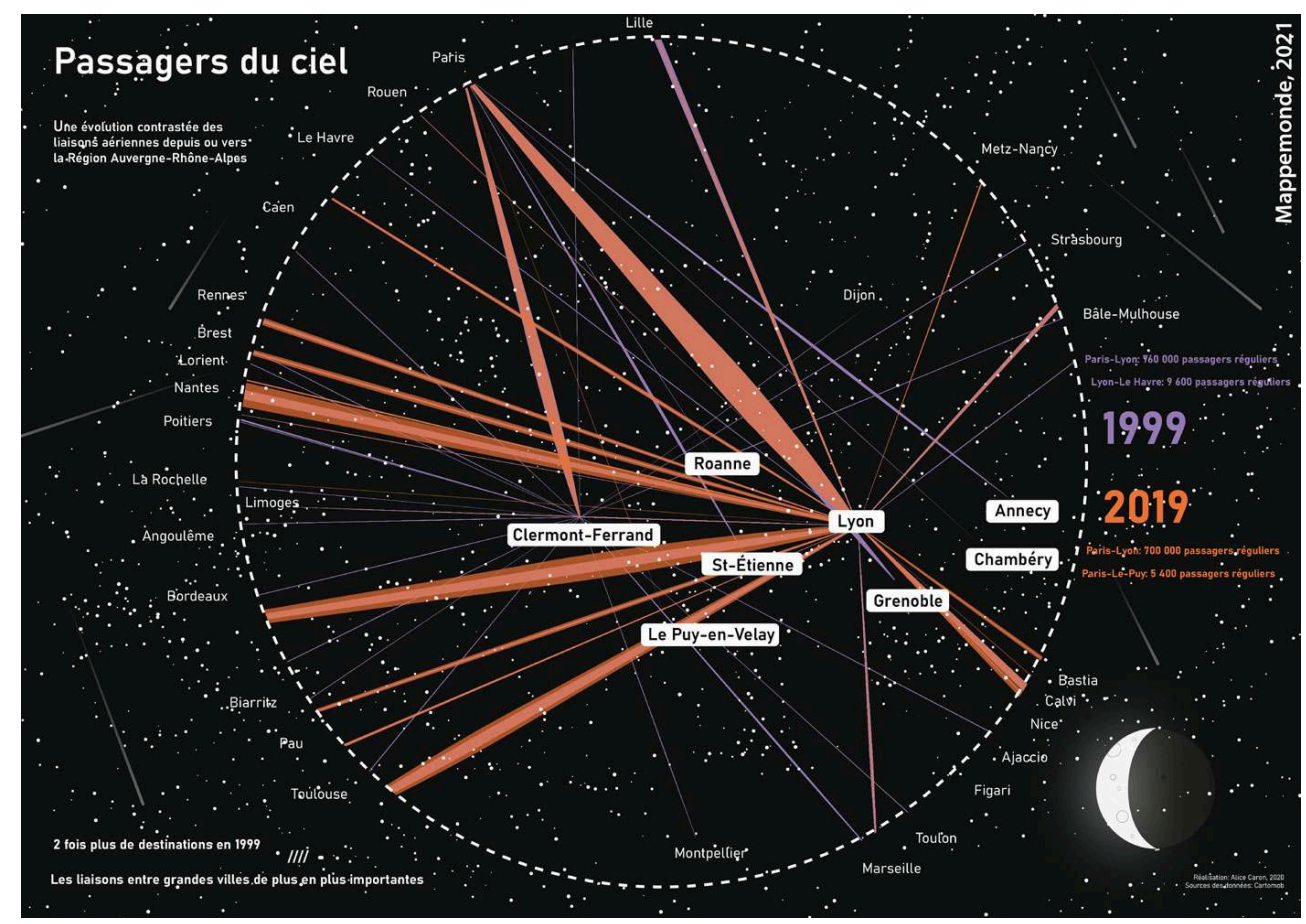


2 Entre prise de conscience écologique et rentabilité économique, la suppression des liaisons jugées périphériques au profit du surdéveloppement des hubs principaux est devenue la règle dans le domaine du transport. Notre production exploite les données de déplacement de personnes par voie aérienne aux années 1999 et 2019. Les liaisons visibles sur la carte ne sont pas exhaustives : nous avons décidé de filtrer les flux en provenance ou/et à destination exclusive des villes de la région Auvergne Rhône-Alpes. Au regard de ce territoire, les possibilités de liaisons aériennes ont presque été divisées par deux en vingt ans. Si, en 1999, il était envisageable de prendre l'avion pour voyager entre Clermont-Ferrand et Dijon, aujourd'hui nous serions amenés à prendre le train, le car ou bien la voiture. Cet appauvrissement dans la pluralité des connexions aériennes s'accompagne de la diversification des types de mobilités, choisis ou subis, par les utilisateurs.

3 Le privilège croissant accordé aux flux aériens dominants est démontré par notre visuel : le linéaire violet, décrivant les liaisons en 1999, est plus éclaté, mais aussi moins large ${ }^{1}$, que le linéaire orange rendant compte de l'année 2019. Le choix de ces couleurs provient de leur complémentarité en terme chromatique et de leur neutralité symbolique $^{2}$. Ces deux périodes du trafic aérien de la région ARA sont donc aisément distinguables l'une de l'autre, sans induire une dichotomie. À l'image des données Eurostat compilées par l'équipe organisatrice du Mapathon, aucun sens n'est donné à la liaison, puisque le volume des flux est bilatéral entre les deux aéroports. Nous avons opté pour conserver graphiquement l'idée d'origine et de destination, en amincissant symétriquement les liens aux extrémités, sans toutefois leur donner une orientation (au moyen d'une flèche, par exemple). La superposition des deux couches de tracés linéaires, décrivant respectivement l'année 1999 et l'année 2019, nous permet de comparer les parcours aériens, tant en termes de volume que de diversité. C'est grâce à une réduction de l'opacité que la superposition des liens va créer une troisième teinte, indiquant la permanence du parcours aérien entre ces périodes. A contrario, les traits orange ou violet appuient sur les mutations, qui paraissent ici structurantes et qui traduisent l'évolution des usages de la mobilité.

4 Nos hypothèses concernant une surconcentration sur certaines liaisons du trafic aérien n'ont pas toutes été validées par le travail statistique associé à la cartographie. Ainsi, une liaison Le-Puy-en-Velay/Paris a été créée entre les deux dates et présente presque deux fois moins ${ }^{3}$ de passagers réguliers que la liaison la moins importante de l'année 1999. Autre résultat contre-intuitif, le flux dominant sur les deux années (ParisLyon) a vu décroître de 60000 son nombre de passagers réguliers en vingt ans. Ce phénomène s'explique toutefois par l'évolution de la ligne ferroviaire connectant les deux métropoles.

5 Conditionnée par mon parcours de cartographe d'édition, j'ai souhaité proposer une carte statique. J'ai construit ce visuel comme un poster à afficher, tant pour son esthétique que pour son aspect pédagogique. C'est pourquoi la mise en page est relativement sobre, quelques phrases viennent appuyer la lecture de la carte, la légende n'est pas académique, mais parfaitement intégrée au reste du document.

6 J'ai été graphiquement inspirée par les planisphères célestes ${ }^{4}$ produits à la fin du $\mathrm{XIX}^{\mathrm{e}}$ siècle. Ces planches dessinées proposent une représentation sphérique du ciel: concernant la production technique, j'ai, dans un premier temps, réalisé des analyses et des traitements statistiques préliminaires dans Excel. J'ai ensuite procédé à un import 
dans l'outil Qgis pour produire les liens ${ }^{5}$ dans l'espace, puis finalisé le graphisme et la mise en page au moyen d'Adobe Illustrator.

7 Pour que le public puisse s'imaginer allongé dans l'herbe à contempler ce spectacle, j'ai opté pour une sémiologie axée sur les marqueurs de l'imaginaire céleste : un fond noir, des étoiles, etc. Je trouvais pertinent le fait de rendre visible la trace complète des avions, car depuis le sol nous ne percevons qu'une portion éphémère de leur trajet sans distinguer la complexité du réseau aérien.

\section{NOTES}

1. La largeur des liaisons transcrit le nombre total de passagers réguliers sur une année, transporté entre les deux aéroports. Nous avons procédé à une classification par seuil naturel (Jenks) et produit cinq classes.

2. À l'instar du rouge ou du vert, par exemple.

3. 5400 contre 9600 pour la liaison Lyon-Le Havre en 1999.

4. Par exemple : https://gallica.bnf.fr/ark:/12148/btv1b53156373g/f1.item

5. Il y a eu des modifications dans le traitement des données une fois celles-ci visibles géographiquement. La première sélection des flux émane d'un choix esthétique, sémiologique, mais vise aussi, et surtout, à transmettre une information claire aux lectrices et lecteurs. 